International Journal of Engineering \& Technology, $7(4.5)(2018) 497-500$
SPC
International Journal of Engineering \& Technology
Website $: \frac{w w . s c i e n c e p u b c o . c o m / i n d e x . p h p / I J E T}{2}$
Research paper

\title{
A way to support the transformation methods for the data in a semantic network
}

\author{
Viacheslav E. Wolfengagen ${ }^{1}$, Larisa Yu. Ismailova ${ }^{1}$, Sergey V. Kosikov ${ }^{2}$ \\ ${ }^{1}$ National Research Nuclear University "MEPhI" (Moscow Engineering Physics Institute), Moscow, 115409 RF \\ ${ }^{2}$ Institute for Contemporary Education “JurInfoR-MGU”, Moscow, 119435 RF \\ *Corresponding author E-mail: jir.vew@gmail.com
}

\begin{abstract}
The paper considers the problem of building of the transformable object-relation mapping. It is shown that an essential part of the task is to get the conversion of data objects doing their representation adjusted for the corresponding data model. It is offered to receive the decision by a semantic method in case of which the formal models of object system and relational system are considered and their interpretations are set. The transformation mappings are considered as a kind of mappings saving interpretations of the given form. Creation of model of converting of data objects on the basis of applicative computing systems is offered what allows to build models of both object, and relational systems dipping in applicative structures with the given means of expression, in particular, to use a lambda-algebra or a lambda model. On this basis the models can be received allow compositions of means of converting and also determination and check of global restrictions for the changes of data determined by the given set of methods of converting. Achievement of flexibility requires use parameterization of the considered construction, i.e. support of dependence of a set of available methods of interpretation on parameters as which semantic characteristics of processed data appear. The prototypes of constructions of models have been used for informational legal supporting of implementation of the best available technology (or just BAT) in practice in Russia.
\end{abstract}

Keywords: Data Conversion; Lambda Algebra; Lambda Model; Object-Relational Mapping; Transformable Object.

\section{Introduction}

Wide circulation of database systems (DB), on the one hand, and systems of the object-oriented analysis, design and programming, on the other hand, stimulates interest in development of means of their automated coordination. Creation of object-relational structures of mapping is represented to one of perspective methods. However, maintenance of mappings, which are scattered in a program code often, requires the considerable efforts and carries to errors, especially if the DB scheme is rather big and intended for service of several systems. Therefore interest in methods of automation of support mappings of the specified kind is clear.

Both object and relational approach can be described in terms of different interpretations of processed data. Interpretations include both representation of data, and representation of the processing procedures, and also means of expression of the restrictions superimposed on data that in terms of the theory of a DB corresponds to a classical concept of a data model.

Establishment of object-relational mapping corresponds to establishment of correlations of the appropriate interpretations. In the most general setting, this task represents version of the task of establishment of equivalence of algorithms and therefore is algo- rithmically undecidable task. Therefore, the specifications of the task allowing to select rather wide classes of special cases of es- tablishment of the considered mappings are of the considerable interest. The method of data interpretation is one of their properties, and as such can be conceptualized and processed in terms, used in general processes of the data characterization. To increase the flexibility of the interpretation control system the essential meaning acquire the possibilities to establish links between the characteristics of the data interpretation (in particular, the rights for access for dif- ferent classes of users) and other characteristics, obtained as a result of data analysis, their classification and so on. The use of various methods and the criteria of classification, including those that are dynamically computed, provides the opportunities of flex- ible interpretation control.

The restrictions of interpretation ultimately are determined by the meaning of observed data that allows include the task of managing data interpretation into the common context of the development of methods of data processing semantically oriented. In this area the methods of conceptual modelling are acknowledged to be good [11]. The inclusion of control of interpretation means into a common conceptual model of data provides the fundamental possibility of taking into account the semantic data characteristics when solving the task of interpretation control, which, in its turn, can count on getting rather flexible methods of building of object- relational mapping.

The semantic characteristics of data, when organizing the interpretation of them, can be accounted in different way. It is possible to bind specific means of data interpretation control with conceptual classes, singled out in models, with frames and other conceptual essences. On this way, in particular, conceptual models can be built similar in features to classic means of object-oriented model- ing [18]. It is also possible to use the model parameterization with the definition of tools for manipulating data, depending on the parameter or sets of parameters

Various methods of interpretation can also be connected with the peculiarities of the data model chosen for the presentation of data within a common conceptual model. For example, in the case when the classical relational model is used the main structure is relation. Accordingly, data interpretations are expressed as expres- sions 
over the relations or their structural elements (e.g., columns): limits of visibility as formulas for determining the apparent rela- tions, and limitation of manipulation in the form of restrictions or any other specialization of operations of relations manipulation.

Specific problems arise when working with the data within the Web-technologies [6]. Web-systems represent one of those classes of applications in which the data may be received from multiple sources. At the same time both the syntax characteristics of data and their semantic structure, generally speaking, are not harmo- nized with each other. The syntactic features mean not only the format of the data (for example, the HTML), but also other cha- racteristics specified in the framework of the used format (e.g., in the case of submission of a collection of articles the authors of a separate article can be named before the title of the article, or after it, and so on). In addition, the data may be irrelevant or insuffi- ciently relevant to solve the task for which they are selected. The problems, appeared in this case, may be interpreted as limitation of interpretation of a specific type.

The rest part of the paper is organized as follows. The Section 2 defines the task of development of conversion of data object model. The Section 3 discusses approaches to the conversion of data object model and to the control of data interpretation depending on the characteristics of their organization by transformable mapping when it is possible. The Section 4 contains the problem definition (model of data interpretation). The lambda-algebras and lambdamodels approach is given. The Section 5 discusses the prototype system supporting the object-relation mapping in a semantic network. In conclusion there is a brief discussion of the obtained results and the prospects of further work.

\section{The task of development of conversion of data object model}

The semantic network will be regarded as consisting of concepts and frames. We'll consider the primary concepts, among which we will highlight the general concepts and constants, and deriva- tive concepts derived from the (previously defined) concepts and frames with the help of the operation of definition. Among the frames we'll allocate frames simple and complex frames received from the simple ones using operations with frames. The methods for determining the semantic network, including its formal model, are defined in details in $[18,6]$.

The semantic network allows to describe data and data collections, and also to highlight their semantic characteristics, that is done on the basis of a certain type of frames (characteristic frames). These frames allow to link the general concepts that describe some of the classes of data, selected in the subject area, with concepts that describe sets of values of their characteristics, and to connected the specific data (presented in a semantic network as constants) with specific values of characteristics.

One of the basic operations with the semantic network is an operation of determination, which allow to consider the expressions, specified by the combination of constructions of semantic network, as atomic, i.e., similar to the primary ones. This operation can be considered as a variant of the abstraction of structures of semantic network. The network as a whole at the same time rece- ives a character of multiply nested structure.

The substitution operation may be considered as the reverse operation; such operation allows determining an object of the semantic network, correlating with the concept, considered as a place for substitution (concept - variable). The substitution, thus, defines the specialization of the semantic network by imposing restrictions on the values of some set of vortexes of network. It is essential that not only atomic object may be substituted, but also a composite object. The essential characteristic of the semantic network of the considered type is the possibility of its nesting in the applicative computing system. This nesting makes it possible to calculate the semantic characteristics by determining the mapping value or the evaluating map. The result of calculation - value - can also be represented as an object of a semantic network.
The applicative nature of the network provides, in particular, for the possibility of network processing by means of the network itself, i.e., self-applicability of the network. Depending on the choice of comprehensive applicative computing system greater or lesser degree of self-applicability might be possible. In particular, it is possible to apply the means of determination of semantic characteristics and the appropriate means of classification to the structures of the network.

On this basis it is possible to determine the semantic structure, which provides a description of possible methods of access to the value contained in the semantic network, and methods of manipulation of that value. Technically, such structure may present a concept that describes a set of frames that define the means of access and manipulation. This design, however, by itself does not provide flexible methods of data transformations, and can only be regarded as a basic building block for the description of corres- ponding instrumental means.

To achieve flexibility requires parameterization of the considered structure, i.e., provision of dependence for a set of the available transformation methods on the parameters, which are mainly the semantic characteristics of the data being processed.

\section{Related work}

The DB modeling connected to a method of management of projects, which is applied to software development, is well known and division of tasks into short working stag- es with overestimations and adaptation of plans $[2,10]$ characterize it. These technologies as a rule are applicable for simulation of databases with use of extensions of UML. They, mainly, are based on the class diagrams presenting data models in the form of in advance known set of stereotypes. In recommendations of Object Man- agement Group (OMG), there are also similar sentences for repre- sentation of data modeling [13].

Mapping from object-oriented representation into relational is known as ORM. However, boundaries of applicability of ORM, possible combinations of the known sample project decisions [2][6], [8], [9], [13] [15], [20] are not analyzed.

Integration aspect of ORM is partially studied in [1] where the system and ENORM technology, which, allegedly, provides plat- form independence, are offered, but has a binding to UML. The EntityFramework platform offers the EDM model based on re- presentation of EER [6], which is expanded treats entities. The EDM model is oriented for needs of multiple objective conceptual simulation for different mechanisms of stability with use of the

.NET platform. Sentences in ENORM [1] consider the general templates of design of ORM better, but do not give the finished decision of the task.

From the known approaches to the analysis of conversion, it is necessary to mark results on transformation of a "speckled" pre- image of the diagram using Java Persistence API (JPA) also called MDJPA [16]. Though JPA also is standard, it is bound to the Java platform. Therefore, it includes many things, which are characteristic only of Java.

In model driven architectures (MDA), it is offered to MDA in design process of system to take model [5] as a basis. For effective implementation of approach of MDA for models, it is necessary to provide integration and computability. At the same time becomes possible to automate process of conversion of models to implementable systems [16]. In UML, there are no opportunities required to describe such models. For example, it is interesting to get some possibilities to express stability or features of mapping of classes in the database. Null information about mapping represents an open problem for development of almost necessary conversions of model.

\section{Lambda algebras and lambda models}

\subsection{Applicative structures}


At the beginning, we define an applicative structure.

$\mathrm{M}=(\mathrm{X}, \cdot)$

Is an applicative structure, if "." is a binary operation on X. Applicative structure is extensional if for all $\mathrm{a}, \mathrm{b}$ from $\mathrm{X}$

$(\mathrm{A} \cdot \mathrm{x}=\mathrm{b} \cdot \mathrm{x})$ implies $\mathrm{a}=\mathrm{b}$.

(We shall write below "A implies B" if B follows from A).

Let $M=(X, \cdot)$ be an applicative structure. Set of terms over $M$ is defined as follows:

i) If $\mathrm{x}$ is a variable then $\mathrm{x}$ is a term over $\mathrm{M}$.

ii) If a $\varepsilon X$ then $c_{a}$ is a term over $M$.

iii) If $\mathrm{A}, \mathrm{B}$ are terms over $\mathrm{M}$, then $(\mathrm{AB})$ is a term over M.There is no other terms over $\mathrm{M}$.

We define an evaluation $\rho$ in $\mathrm{M}$ as a mapping from variables to $\mathrm{M}$. Let $\mathrm{M}=(\mathrm{X}, \cdot)$ be an applicative structure. Interpretation of a term in $\mathrm{M}$ with the evaluation $\rho$ is defined as follows.

i) $[X]_{\rho}=\rho(\mathrm{x})$.

2. $\left[c_{a}\right]_{\rho}=a$.

ii) $[\mathrm{AB}] \rho=[\mathrm{A}] \rho \cdot[\mathrm{B}] \rho$.

The definition of an applicative structure is too general for an adequate modelling of the specific problem areas. So it is interest- ing to consider applicative structures with different restrictions. The strong enough and useful restriction is combinatory com- pleteness. Let $\mathrm{M}=(\mathrm{X}, \cdot)$ be an applicative structure and $\varphi: \mathrm{X}^{\mathrm{n}} \rightarrow \mathrm{X}$ be a mapping.

1) $\varphi$ is representable over $M$, if there exists $f \varepsilon X$ such that for all $\mathrm{a}_{1}, \ldots, \mathrm{a}_{\mathrm{i}}, \ldots, \mathrm{a}_{\mathrm{n}} \varepsilon X \mathrm{Xf} \mathrm{a}_{1} \ldots \mathrm{a}_{\mathrm{n}}=\varphi\left(\mathrm{a}_{1}, \ldots, \mathrm{a}_{\mathrm{n}}\right)$

2) $\varphi$ is algebraic over $M$, if exists such term $A$ over $M$ that $F V(A)$ $<\left\{\mathrm{x}_{1}, \mathrm{x}_{\mathrm{n}}\right\}$ and for all $\mathrm{a}_{1}, \mathrm{a}_{\mathrm{i}}, \mathrm{a}_{\mathrm{n}} \varepsilon \mathrm{X}$

$\Phi(\mathrm{a} 1, \mathrm{an})=[\mathrm{A}] \rho(\mathrm{x}:=\mathrm{a})$

It is obvious that all representable functions are algebraic. Applicative structure $\mathrm{M}=(\mathrm{X}, \cdot)$ is combinatory complete if all functions algebraic over $\mathrm{M}$ are representable over $\mathrm{M}$. Combinatory algebra is an applicative structure $\mathrm{M}=(\mathrm{X}, \cdot, \mathrm{k}, \mathrm{s})$ having selected elemens $\mathrm{k}$, $\mathrm{s}$ that satisfy the following equation

$K \cdot x \cdot y=x$

$\mathrm{S} \cdot \mathrm{x} \cdot \mathrm{y} \cdot \mathrm{z}=\mathrm{x} \cdot \mathrm{z} \cdot(\mathrm{y} \cdot \mathrm{z})$

We shall omit below the mark of - operation when it is obvious.

\subsection{Lambda algebras}

It is possible to model a lambda abstraction in a combinatory algebra $\mathrm{M}$ using $\mathrm{k}$ and $\mathrm{s}$. Therefore, it is possible to interpret lambda terms in $\mathrm{M}$. Let $\mathrm{C}$ be a set of constants. We denote as $\Lambda(\mathrm{C})$ a set of lambda terms (possibly) containing constants from $\mathrm{C}$. If $\mathrm{M}=(\mathrm{X}, \cdot$, $\mathrm{k}, \mathrm{s})$ is an applicative structure then $\Lambda(\mathrm{M})$ is by definition $\Lambda(\mathrm{X})$. Let me be a combinatory algebra. We consider mappings $C L \Lambda(\mathrm{M})$ $\rightarrow \mathrm{T}(\mathrm{M})$

$\Lambda: \mathrm{T}(\mathrm{M}) \rightarrow \Lambda(\mathrm{M})$

Defined as follows:

$\mathrm{XCL}=\mathrm{x} \mathrm{CCL}=\mathrm{c}$

$(\mathrm{MN})_{\mathrm{CL}}=\mathrm{M}_{\mathrm{CL}} \mathrm{N}_{\mathrm{CL}}$

$(\Lambda \mathrm{x} . \mathrm{M})_{\mathrm{CL}}=\lambda^{*} \mathrm{x} . \mathrm{MCL}_{\mathrm{CL}}$

And

$\mathrm{x}_{\Lambda}=\mathrm{x}$

$\mathrm{K}_{\Lambda}=\lambda \mathrm{xy} . \mathrm{X}$

$\mathrm{S}_{\Lambda}=\lambda \mathrm{xyz} . \mathrm{xz}(\mathrm{yz}) \mathrm{c}_{\Lambda}=\mathrm{c}$

$(\mathrm{MN})_{\Lambda}=\mathrm{M}_{\Lambda} \mathrm{N}_{\Lambda}$

For $\mathrm{M}, \mathrm{N} \varepsilon \Lambda(\mathrm{M})$ we suppose by definition $[\mathrm{M}]_{\rho}=\left[\mathrm{M}_{\mathrm{CL}}\right]_{\rho}$

$\mathrm{M}, \rho \vDash \mathrm{M}=\mathrm{N}$ if and only if $[\mathrm{M}]_{\rho}=[\mathrm{N}]_{\rho}$

$\mathrm{M}=\mathrm{M}=\mathrm{N}$ if and only if $\mathrm{M}, \rho \neq \mathrm{M}=\mathrm{N}$ for all $\rho$

We define lambda algebras as combinatory algebras $\mathrm{M}$ where $\mathrm{A}_{\Lambda}=$ $\mathrm{B}_{\Lambda}$ implies $\mathrm{M}=\mathrm{A}=\mathrm{B}$
For all $\mathrm{A}, \mathrm{B} \varepsilon \mathrm{T}(\mathrm{M})$

\subsection{Lambda models}

We call a lambda model such lambda algebra that the following condition is satisfied:

For all $\mathrm{x}(\mathrm{an} \mathrm{x}=\mathrm{b} \mathrm{x})$ implies one $\mathrm{a}=$ one $\mathrm{b}$, where $1=\mathrm{S}(\mathrm{KI})$.

Let me be a combinatory algebra. Then $1 \mathrm{ab}=\mathrm{ab}$.

If $\mathrm{M}$ is a lambda algebra then

One $=\lambda \mathrm{xy}$. xy, and so $1 \mathrm{a}=\lambda \mathrm{y}$. ay;

One $(\lambda \mathrm{x} . \mathrm{A})=\lambda \mathrm{x}$. A, for all a $\varepsilon \mathrm{T}(\mathrm{M}) ; 11=1$.

Proposition. $M$ is a lambda model if and only if $M$ is a weak extensional lambda algebra.

Proof. 1. Let Me be weak extensional. So for all $\mathrm{x}$

$($ An $\mathrm{x}=\mathrm{b} \mathrm{x})$ implies $\lambda \mathrm{x} . \mathrm{a} \mathrm{x}=\lambda \mathrm{x} . \mathrm{b} \mathrm{x}$ implies $1 \mathrm{a}=1 \mathrm{~b}$.

2. Let Me be a lambda model. So

For all $\mathrm{x}(\mathrm{A}=\mathrm{B})$ implies

For all $x((\lambda x$. A $) X=(\lambda x$. B $) x)$ implies

$[1](\lambda x . A)=[1](\lambda x . B)$ implies $\lambda x . A=\lambda x . B$

In practice, it is possible to offer a general method of building the lambda algebra and even the lambda model containing given partially ordered set. The necessary requirements on partial order are rather weak. So it is possible to include in this partial order the given set of relations, thus modelling the data in relational DB. High-ordered functions then can be used to model the operations on relational DB. These functions are represented in lambda mod- el as applicative objects, so they can be regarded as a part of ob- jectrelational mapping.

\section{Prototype system of support to the semantic network}

The testing of the proposed ways to implement the transformation procedures requires means of support to the semantic network. The development of such means is in the initial stage at present. The group of authors has implemented the prototype of tools to support the network with the opportunities of the object-relation mapping, which is currently at the stage of a comprehensive de- bugging. The system provides the following: definition of concepts, including general concepts and constants; definition of frames, including primary frames and derivative frames based on concepts; substitution of a fragment of the semantic network, presented in the form of a correct expression of language for defining the network, on the place of substitution, specified by the variable of frame; de- termination of the context of computation to clarify the interpreta- tion of the frame; computation of the value of expression of a semantic network and it's mapping in the system-a certain form.

The architecture of the prototype system includes the following:

1) parsing module, which provides (among others) the identifica- tion and analysis of structures of semantic networks with assigned appropriate types to them for application of transformation me- thods;

2) Module for storage of the semantic networks structures and access to them for applying the specified;

3) module of ex- pression generating, signifying the construction of the semantic network;

4) module of generation of auxiliary XML-constructs, used to represent the values;

5) module of computation of values. Since the transformation methods are the objects "first order" for the system, obtained from the underlying lambda model, their signifying is carried out at the expense of the common computa- tional tools of the system. In particular, one can search for policies by semantic characteristics with its subsequent signifying. The testing of the elements of the transformation methods implementation was carried out with the help of the legal tasks using the data presented in XML dialect. The testing allowed to make a conclusion about the applicability of methods to support the data inter- predation within the bounds provided by 
the given implementation of the supporting system. The prototypes of constructions of models have been used for informational legal supporting of implementation of the best available technology (or just BAT) in practice in Russia.

\section{Conclusion}

The paper researched the task of supporting the control of data interpretation with regard to their semantic characteristics. The semantic characteristics were attributed to the data with the help of the semantic network, which can be embedded in the computing system of the applicative type. The following results were obtained: the approaches to the problem of an data interpretation management using semantic information have been analyzed; the definition of the transformation methods was offered and the basic tools to work with them has been described; the task to support the transformation methods for the data in a semantic network has been set; the variants of determination of structures of semantic networks providing the support to transformation methods have been considered; the method of implementation of the policy sup- port mechanisms in the semantic network has been offered; the possibility of implementation of tools supporting the transforma- tion methods in the semantic network has been briefly considered. At present time the authors discuss the work in the sphere of the definition of transformation methods to the semantic network. Promising areas for further work include the development of the following: mathematical models of the semantic network in their connection with the task of the transformation support; models of transformation methods that provide the description of data inter- pretation management; architecture and support system of the semantic networks, providing data interpretation management based on transformation methods.

The successful solution of problems encountered can be achieved, in particular, by the methods of applicative computing systems. As expected, the semantically oriented systems of data transformation that provide flexible control of data interpretation under processing can be obtained as the result.

\section{Acknowledgement}

The research is supported in part by the RFBR grants 17-07-00893, 16-07-00914, 16-07-00892, 16-07-00912,

16-07-00909, 18-07-01082.

\section{References}

[1] Torres A, Galante R, Pimenta M, "ENORM: An Essential Notation for Object-Relational Mapping", SIGMOD Record, June 2014, Vol 43, No. 2, (2014). pp. 24-28.

[2] AUML Profile for Data Modeling: 2003.http://www.agiledata.org/essays/umlDataModelingProfile.html.

[3] Active Record - Object-relation mapping put on rails 2012 http://ar.rubyonrails.org/. Accessed: 2013-10-01.

[4] Atzeni P. et al. "The relational model is dead, SQL is dead, and I don't feel so good myself', SIGMOD Rec. 42, 1 (Jul. 2013), pp. 64 68.

[5] Beydeda, S. et al. Model-Driven Software Development. Springer, (2005).

[6] Blakeley JA et al. "The ADO.NET entity framework: making the conceptual level real". SIGMOD Rec. 35, 4 (Dec. 2006), pp. 32-39.

[7] Conceptual Modeling. Proceedings of 35th International Conference by ed. Comyn-Wattiau, I. et al, ER 2016, Gifu, Japan, November 14-17, 2016, LNCS, Volume 9974 (2016), 2016, Springer.

[8] Elmasri R \& Navathe SB, Fundamentals of Database Systems, Addison Wesley, (2003).

[9] Essential ORM Modeler: 2013. http://sourceforge.net/projects/ eorm/.

[10] Gabriella Castro Barbosa Costa, "Using data provenance to improve software process enactment, monitoring and analysis", Proceedings of the 38th International Conference on Software Engineering Companion, ICSE '16, pp. 875-878, New York, NY, USA, 2016. ACM.
[11] Ismailova L, "Criteria for computational thinking in information and computational technologies", Life Science Journal, 11(9s): pp. 415 420, November 2014

[12] OMG 2001. OMG's Model Driven Architecture.

[13] OMG 2005. Request for Proposal Information Management Metamodel (IMM)

[14] Population Modeling Working Group. "Population modeling by examples (wip)", Proceedings of the Symposium on Modeling and Simulation in Medicine, MSM '15, pp. 61-66, San Diego, CA, USA, 2015. Society for Computer Simulation International.

[15] SQL Alchemy - The Database Toolkit for Python: 2012. http://www.sqlalchemy.org/.

[16] Torres a et al. "A synergistic model-driven approach for persis- tence modeling with UML", Journal of Systems and Software. 84, 6 (Jun. 2011), pp. 942-957.

[17] Torres, A. et al. "Technical Report - Comparing ENORM and separated modeling using Relational and UML class models: a withinsubjects experimental study", http://www.inf.ufrgs.br/ atorres/ sig$\bmod 2013 /$

[18] Wolfengagen, VE. "Semantic Modeling: Computational Models of the Concepts", Proceedings of the 2010 International Conference on Computational Intelligence and Security (CIS '10), IEEE Com- puter Society, Washington, DC, USA, pp. 42-46.

[19] Wolfengagen, VE et al. "Migration of the Individuals", Procedia Computer Science, Volume 88, 2016, pp. 359-364 http://dx.doi.org/10.1016/j.procs.2016.07.449.

[20] UML $2.4 .1 \quad$ Superstructure: http://www.omg.org/spec/UML/2.4.1/Superstructure/PDF.
2011. 